\title{
Research and Education
}

\section{Medical Informatics and Telemedicine at the Politecnico di Milano}

\begin{abstract}
Research and training programs in Medical Informatics and Telemedicine offered at the Politecnico di Milano are summarized. The educational pathways refer to the programs of: the (5-years) Laurea Degree, the (3-years) Diploma Universitario Degree, the post-Laurea (3-years) Doctoral Degree, the (1-year) Master's Degree, and a Continuing Education Program. The research programs focus on databases and extend to optimization of the "Visible Human Dataset-Milano Mirror Site" services. Additional activities relate to standardization at both the Italian and European levels, and to cooperation with hospitals and manufacturers.
\end{abstract}

Keywords: Education, Databases, Visible Humans

\section{Framework}

One generation ago, at the beginning of the 1970s, we were a small group of enthusiastic and idealistic young persons, fascinated by the ideas of the "mission" of Biomedical Engineering for the benefit of mankind. Since that time, we have always been too many persons in relation to the number of academic positions available: this remains true today. This was also evident in the Academic Year 1997/98, when we gave the opening pddress to the 236 freshmen enrolled for the five-year Laurea Degree in Biomedical Engineering. However, despite having been - academically foo many at one time, we believe that we are still too few in relation to the need of our skills in Italy today. This is probably the fuel that maintains our enthusiasm, one generation later. According to the ideas and the behavioral tyles generated by our father in scisnce, Emanuele Biondi (still active and creative today) and according to our ometimes conflicting scientific beliefs, we forged the Department of Bio- medical Engineering of the Politecnico di Milano. One can find us at http:// www.biomed.polimi.it. The Department, founded in 1990 and now chaired by Antonio Pedotti, is also the main academic focus for the educational and research activities offered by the Politecnico di Milano in the field if Medical Informatics and Telemedicine (also described at websitehttp://www. medinfopoli.polimi.it).

\section{The Educational Activities}

The inherent aim in each educational program offered in Medical Informatics and Telemedicine at the Politecnico di Milano is to enable students to acquire technical skills in order to become information and communication technology experts, in whom the physician believes. Many educators know how difficult it is to achieve such an aim, but there are no alternatives. This has been proven by the history of technology in medicine.

A target such as this requires that students personally acquire some fun- damental roots of knowledge. The first root relates to the problems in the fields of Medicine and Health Care. The knowledge should be deep, even though theeducational traditions may beyoung. It should avoid excessive hope in the ability of the physician, as the client of the products developed for him, to provide a detailed list of the activities required to solve his problems. The second root of knowledge required relates to Information and Communication Technology tools. Methods, techniques, devices and systems, suitable for implementing the solutions for the problems present in Medicine and Health Care, should be carefully selected.

The envisaged working relation foresees two interactive but separate roles. The physician plays the customer role: he asks for solutions, and evaluates their implementation. The medical informatician plays the provider role: he has to develop the solutions. The overall relation will probably lead to a permanent conflict and interactive negotiation between the two roles. Nevertheless, they have to share the posi- 
tive final aim of the conflict, i.e. the development of solutions for the benefit of patients. Evolution brings additional difficulties. We refer to the evolution of the needs of Medicine and Health Care on the one side, to the very fast evolution of Information and Communication Technology tools on the other side, and to the fact that neither may be well aligned to the needs of Medicine. For the Medical Informatician to remain in good dialogue and collaboration with the physician is a difficult but necessary task. The different educational curricula and post-degree programs offered at the Politecnico di Milano are devised to fulfil this aim.

\subsection{The Laurea Degree}

At this level, the concept of "medical information and communication technology, reliable interlocutor for the client physician (MICTRID)" implies that the medical informatician will cooperate with physicians at top management levels. Examples of typical activities are the choice of new trends in hospital information systems (will we go with Java?), and the tools for achieving data security and privacy (does PCASSO make sense for us?), etc. Professional roles such as junior advisor, senior programmer and systems manager are targets within the MICTRID concept.

The announcement of the 1996/97 first-semester edition of the course on Medical Informatics is mentioned in Table 1. The course is now offered to students of the five-year "Laurea" degrees in Biomedical Engineering, Computer Engineering, Electronic Engineering and Mechanical Engineering. Prerequisites for admission are two basic courses in computer programming, taken in the first and second year of the "Laurea" degrees. Additional information on teaching and research activities at the Laboratory of Medical Informatics is available at our web site,http://www.medinfopoli. polimi.it. In addition, for $1997 / 98$, a "learning by doing" project, will be developed (at an Internet-connected classroom), entitled "Internet for Medicine and Health Care". Its final products will be available as MedInfoPoli'98 on CD/WEB.

\subsection{The Diploma Universitario Degree}

At this level, the MICTRID concept will involve cooperation between the medical informatician and physicians at the daily practice levels. Examples of typical activities are the optimal maintenance of systems and devices, minoradditional customization implementations, new releases, customer support, etc. Professional roles such as junior programmer, product manager, systems administrator are targets within the MICTRID concept .

Within the framework of the Diploma Universitario Degree in Biomedical Engineering, the Medical Informatics course is compulsory for all students in their third and final year of attendance. It comes after the single course in Basic Computer Programming taken in their first year. The addition of a second course in the field, to be taken before Medical Informatics, would be welcome; however, the large number of subjects given to the students has precluded this up to now. The establishment of a "Hospital Information Systems" module within the program is now under evaluation. This could be an effective solution for delivering the necessary concepts to the students. Additional information on the Diploma Universitario Degree in Biomedical Engineering is available at http://www.biomed.polimi.it.

\subsection{The Post-Graduate Master's Program in Telemedicine}

The typical activities within the MITRID concept outlined for the Laurea Degree level are also valid for this postgraduate one-year program. The target audience, however, is mainly those Laurea who graduated in Engl neering but did not take courses in Medical Informatics and Biomedic Engineering, and those Laurea wh graduated in Medical Sciences, Phan maceutical Sciences and, Biologict Sciences, who did not take courses in Information and Communication Tech nology. Professional roles such as jun, ior advisor, senior programmer, sys, tems manager, professional advisoll are within the MICTRID concept .

The 1997/98 edition of the Master 1 Program in Telemedicine is open to 15 candidates. Entirely sponsored this year by the Lombardia Region local gov ernments, the Master's Program in Telemedicine comes from the Dipartimento di Bioingegneria and the Cefriel Consortium. Among the several consortia managed by the Politecnicodi Milano, the Cefriel Con sortium has offered a Master's course in Information Technology for the last 10 years. Additional information is available at the web site of the Cefrie Consortium, athttp://www.cefriel.it

\subsection{The Post-Graduate Doctorat Program in Bioengineering}

At this level, the MICTRID concen focuses on specific research subjects Examples of activities are modelin and implementing users views by object-oriented approaches, applying object-oriented technologies in modeling and querying temporally-oriented clini cal databases, etc. These students interact with physicians involved in research and submit results for publica tion in renowned scientific journale Professional roles such as projec leader, scientific advisors, research are targets within the MICTRID com cept .

\subsection{The Continuing Education Program}

The Engineering Faculty of the Politecnico di Milano offers a Contint ing Education Program which consis of some 50 courses (of varying dura 
ion) covering many of the engineering disciplines. The program, recently renamed "Poliedra", is described athttp:/ Awww.polimi.it. For Medical Informatics and Telemedicine, the topic Internet for Medicine and Health care" is offered as a short laboratory course, inspired by the concept of tearning by doing".

At this level, the MICTRID concept involvescooperation between the medical informatician (frequently already working at an institution), and the institution's physicians and managers for rapid application of the benefits of some services available via Internet. Examples of typical activities are use of web addresses in relation to medical publishers and scientific associations, methods fordistributing medical guidelines, systems for connecting GPs to the hospital specialist, etc. The targets within the MICTRID concept relate to the need to acquire updated knowl- edge on recent advances in Medical Informatics and Telemedicine.

\section{The Research Scenario}

The research scenario has, of course, been influenced by the described educational framework. The Biomedical Engineering stream has inspired the construction of the "Politecnico Biosignals Archives" described here below, now available both on CD-ROM and at http:// www.cilea.it. Later, interest focused increasingly on advanced clinical databases and on features such as the integration of data and images, and the temporal characteristics of data. Most 'of the research is included in the activities program of the Centro di Ingegneria Biomedica, a cooperative center of the Italian National Research Council with the Politecnicodi Milano, now chaired by Ferdinando Grandori and located at the Dipartimento di Bioingegneria, described athttp://www.biomed.polimi.it.

\subsection{Recent Research Activities}

\subsubsection{Textual and image data integration - ARCADIA}

Use of database techniques to store medical records has existed for more than forty years; however, some aspects remain unresolved. This includes management of textual data and image data within a single database. Objectoriented techniques applied to a database management system (DBMS) allow the definition of suitable data structures (e.g. to store digital images): some facilities allow the use of predefined structures when defining new ones. Currently available objectoriented DBMS, however, still have to be improved, both in the schema update and in the query facilities. We

Table 1. Activities at the Medical Informatics Department of the Politechnico di Milano'

Sections marked with (*) are not in English

\begin{tabular}{|c|c|c|}
\hline $\begin{array}{l}\text { Research } \\
\text { - Rong-te rm subjects } \\
\text { - Recent subjects } \\
\text { Thesis (*) } \\
\text { - Discussed titles } \\
\text { - Candidate subjects }\end{array}$ & $\begin{array}{l}\text { Teaching } \\
\text { - Curricular course program } \\
\text { - for the ( } 5 \text {-year) Laurea Degree } \\
\text { - for the ( } 3 \text { year) Diploma } \\
\text { Universitario Degree }\left(^{*}\right) \\
\text { - Informazioni sul Corso } 1996 / 97\left(^{*}\right) \\
\text { - Information on } 1996 / 97 \text { Course } \\
\text { - Continuing education courses }\left(^{*}\right) \\
\text { - Pictorial home page of the } 1995 / 96 \text { course }\end{array}$ & $\begin{array}{l}\text { Liaisons } \\
\text { - Scientific advice } \\
\text { - Scientific journals } \\
\text { - Scientific and professional associations } \\
\text { - Standardization bodjes } \\
\text { Permanent Cooperation } \\
\text { - Ospedale Maggiore di Milano (Servizio di } \\
\text { In formatica Medica) } \\
\text { - International Heart School } \\
\text { - Accademia Internazionale di Bergamo per } \\
\text { Scienze Mediche Avanzate }\end{array}$ \\
\hline $\begin{array}{l}\text { Personnel } \\
\text { - Present } \\
\text { - Former }\end{array}$ & $\begin{array}{l}\text { Publications } \\
\text { - Submitted manuscripts } \\
\text { - Selected list } \\
\text { - Extended list }\end{array}$ & $\begin{array}{l}\text { Prototypes } \\
\text { - RivaRocci\& Polimi } \\
\text { - ITALICA } \\
\text { - Politecnico Biosignal A rchieves } \\
\text { - ARCADIA } \\
\text { - GCH-OSQL } \\
\text { - PAFHPI } \\
\text { - S-WATCH-QL } \\
\text { - Mallat\&Polimi } \\
\end{array}$ \\
\hline \multicolumn{3}{|c|}{$\begin{array}{l}\text { - Visible H u m an D a taset - M ilano M irror } \mathrm{Site} \\
\text { - Activities and events organized by external bodies } \\
\text { - WWW node design and implementation for Clinical and Health Care services (interactive) } \\
\text { - Continuing education Medical In formatics courses at private and public institutions } \\
\text { - International consortia for submitting grant proposals to the European Union and to other funding agencies (inactive) } \\
\text { - Consulting for Clinical and Health Care Inforamtics systems and services (inactive) } \\
\text { - Special Issue on TIME-ORIENTED SYSTEMS in MEDICINE of the international journal Computers in Biology and Medicine }\end{array}$} \\
\hline
\end{tabular}


developed a prototype of a medical record which includes some multimedia features, managing both textual and image data. The prototype considers data from the medical records of patients subjected to percutaneous transluminal coronary artery angioplasty (PTCA). We developed it on a Sun workstation with Unix operating system and ONTOS ${ }^{\mathrm{TM}}$ as an object-oriented DBMS. The prototype has been named ARCADIA (A Reàlization of a coronary-artery Angioplasty Data and Image Administator).

\subsubsection{A temporal data model for clinical data}

A time-Oriented Medical Record is a medical record based on a temporal data model. In both the clinical and research field there is a need for managing temporal aspects of data in a more functional and complete way in order to satisfy particular needs of that application field. The model we developed has been applied to data from patients who undergo coronary artery angioplasty. These patients, after a 23 day hospitalization, undergo periodic follow-ups aimed at verifying the efficacy of angioplasty. Management of temporal aspects of this data is therefore relevant. The temporal data model has been implemented by means of an Object-Oriented DataBase Management System (OODBMS). Descriptions of the model and of some temporal queries which have been made available by the implementation of this model are provided.

\subsubsection{Window-based object-oriented user interface}

After designing the temporal data model, which has the ability to manage different time granularities of the clinical information, we focused our attention on designing a window-based user interface. We designed a windowobjects hierarchy for the management of data related to PTCA patients. The user interface allows the user to:

- display, enter and modify the clinical data of PTCA patients

- manage more effectively temporal clinical data having different time granularity; more particularly, the user-interface software is able to graphically represent temporal data having different granularities

- manage more effectively textual and image data and their relationships.

\subsubsection{Temporal object SQL}

After developing the temporal clinical data model, we are designing a Temporal Object SQL. The Temporal Object SQL relies on the non-interactive version of OSQL of ONTOS to make queries on temporal data having different time granularities (i.e. time units). Our temporal SQL uses a threevalued logic (true, false, undefined) in order to manage the uncertainty coming from relationships between temporal data at different granularities. The temporal SQL has been implemented by $\mathrm{C}++$ and ONTOS.

We are strengthening the integration between textual and image data into a database. In particular, we are designing a system which is able to integrate more image data coming from different sources: angiographic images having heterogeneous clinical contents, image sequences, echocardiographic images, and images coming from nuclear medicine. We are also making efforts toward integrating textual, image and graphical data to allow a deeper analysis of the collected data.

\subsubsection{Angiocardiographic image compression.}

We defined and implemented three new irreversible compression techniques for digital angio-cardiographic static images: Brightness Error Limitation (BEL), Pseudo-gradient Adaptive Brightness Error Limitation (PABEL), Pseudo-gradient Adaptive
Brightness and Contrast Error Limit? tion (PABCEL). To scan digital im ages we implemented an algorith based on the Peano-Hilbert plane-fir ing curve. We applied our compre sion techniques to 168 static image selected from angiocardiographic 35 mm films. We achieved best compres sion results applying the PABCEI method, obtaining a mean compres sion ratio of about 8:1. Consulted cary diologists did not find significant diag nostic differences between origint images and reconstructed ones.

\subsection{Long-term Research Activities}

3.2.1 - Normal subjects ECG-andrespiration reference databases on CD-ROM

A number of new quantifiers recently suggested for clinical practio and already provided by some mon toring instruments are considered to be insufficiently tested on the normal popuy lation. The CD-ROM based technol ogy allows the construction of easy-tol use dynamic archives of biosignals and bioimages. They can give truly positiv contributions to the needed testing prow cedures. This situation refers to the validation of computer algorithms for ECG analysis. We built the Politecnis Biosignals Archives on CD-ROM where two biosignal databases frodt young normal subjects are stored. The first database is the Politecnico Ca Granda VCG/ECG Database on Young Normal Subject. It was devel oped to investigate unevident ECd variability. It consists of 23 recordin about 30 minutes long, from 23 youn normal subjects. Recordings consistol the three orthogonal ECG leads cof rected according to Frank. A co certed effort was made to ensure th maximum fidelity of recordings. Ma technical features are: sampling fit quency of $500 \mathrm{~Hz}$, resolution of $2.4 \mathrm{u}$ unit on 12 bits. The second database the Poli/Medlav Database on EC 
and-Respiration, developed to investigate interaction among cardio and respiratory systems. It consists of 20 recordings, about 23 minutes long, from 20 young normal subjects. Recording consists of three ECG leads (the three orthogonal leads corrected according to Frank), and two respiratory traces: the respired airflow and the body volume variations, both measured with a plethysmographic chamber. Main techtical features are sampling frequency of $500 \mathrm{~Hz}, \mathrm{ECG}$ resolution of $2.4 \mu \mathrm{V} /$ unit, respiration resolution of about $0.125 \mathrm{~mm} / \mathrm{s} /$ unit, both on 12 bits. Data format respects the practical standards used for similar applications in recent years.

\subsubsection{The DEKG software}

One question to be answered by any teacher relates to ways to help students when they make mistakes. To give such help in the field of teaching dynamic electrocardiography, we built up an effective integration of two CAI tools that we have developed in recent years. The first CAI tool is the DEKGTrainer system, an instructional aid oriented towards environmental simulation and summative evaluation in the field of dynamic electrocardiography teaching. The second CAI tool is the Clinic Arrhythmia Database - a database which holds a teaching-oriented version of the arrhythmia knowledge. The integration we have implemented is the D.E.K.G-Manager system. It provides a formative evaluation of a student who has made classification mistakes. The DEKG-Manager intends, therefore, to correct the erroneous beliefs which could have driven the student to formulate a wrong diagnosis. The system has two working modes. The first mode is dedicated to formative evaluation. When the student classifies a heart beat in a wrong way using the DEKG-Trainer, our system allows the student to compare the morphological characteristics of the beat really present in the tracing with those pertaining to the erroneous diagnosis, highlighting the difference between the two descriptions. The student can also examine the segment of tracing related to a beat classified in a wrong way and analyze the classes of rhythms compatible with the class of the current beat. The second mode consists in the query of the Clinic Arrhythmia Database. In this case, the student can freely compare the different descriptions contained in the classes of beats present in the database and examine the rhythms compatible with them. The descriptive information obtained from the Clinic Arrhythmia Database is displayed within a graphical schema similar to the Entity-Relationship model. Graphical symbols and colors are used to underline a different degree of importance of the morphological attributes typical of each considered diagnostic class. Finally, the DEKG-Manager system, though not all-encompassing, intends to be an effective and easy-to-use aid to a student who wants to fill the gaps in his knowledge of dynamic electro-cardiography.

\subsubsection{The MSx2 software (Multi- \\ Service Medical Software)}

Many clinics are interested in using software packages in daily practice. Such packages, however, if not integrated are seriously limited in their scope. This means that their daily use requires switching from one program to another and, at times, interrupting the running of an individual program. A multi-task approach would not solve the common problem with separate packages, as it does not dispense with the need to input the same data repeatedly. We developed a Multi-Service Medical Software package (MSx2), which was also developed as an example of practical integration of some clinically relevant functions. The package runs on a PC in an MS-DOS environment and integrates a timeoriented medical record management unit (TOMRU) and a drug information management unit (DIMU). TOMRU handles the follow-up data of ambulatory patients. DIMU performs a druginformation reference service as concerns posology, content, effects and possible interactions. Of the possible database configurations allowed by MSx2, the cardiology patient database $(\mathrm{MSx} 2 / \mathrm{C})$ and hypertensive patient database $(\mathrm{MS} \times 2 / \mathrm{H})$ were developed. ConfiguringMS 2 should in any case be left to an expert user, in charge of database management. The clinical information to be included in the configurations was obtained by discussing the needs of and obtaining the consensus of clinical practitioners. MSx2/C was handed over to hundreds of clinical centers during the computerized itinerant courses held to train would-be users. MSx2 can easily transfer patient data to statistical processing packages.

\subsubsection{Self-monitoring of paroxys-mal atrial fibrillation in ambulatory patients}

Paroxysmal Atrial Fibrillation(PAF) is a typical cardiac pathology where both Holter monitoring and traditional ECG are inadequate instrumentation systems. Probability to record PAF events - symptomatic episodes occurring in unpredictable moments - with 24 hours recording is low. Production of an objective instrumental documentation based on ECG for ambulatory patients is still a problem. Documentation might be obtained with a portable instrument given to the patient for self-documentation of pathological events. The device must permit many hours of active recording, even if it stays on standby for months. This is allowed by the Paroxysmal Atrial Fibrillation Histogram-based Portable Identifier (PAFHPI) which we have built. The PAFHPI is a low-cost and easy-to-use portable solid-state recorder. The method used to investigate atrial fibrillation events is based 
on morphology of some histograms: the first based on RR intervals values series and the second based on one of time.

\subsubsection{Public-domain medical}

software evaluation

A vast quantity of public-domain medical software is available. Therefore we developed a classi-fication scheme that includes identification data (e.g., name of the software, author...), description (hardware and software requirements), classification (software category, application domain...) and evaluation data (external and internal quality factors, according to software engineering criteria). We tested the scheme on the public-domain software available at the SCAMC $®$ meetings (about 36 Mbytes). The software was classified by employing students from a medical informatics Masters' course in computer science; the information collected was stored in a database we developed with Paradox.

\subsubsection{Riva-Rocci \& Poli-Mi}

For non-invasive measurement of systolic and diastolic blood pressures values, some instruments allow recording of complete pressure signals. Recent advances in biomedical signal processing have suggested new methods for deeper investigation of such signals, both in the time and the frequency domain. The Riva-Rocci \& Poli-Mi Signal Base Management System is a software package that is easily used by physicians for testing these methods in different pathophysiological conditions on a large-scale basis.

\section{The "Visible Human Dataset - Milano Mirror Site"}

The Visible Human Dataset (VHD) is the largest atlas of multimodal digital bioimages of the human body. A product of the National Library of Medi- cine in Bethesda, it consists of about 20,000 images from selected horizontal sections of the male and the female body. Besides photographic images, there are MRI and CT images. The VHD includes presentation of transverse, sagittal and oblique cross-sectional images of the body, as well as illustrating extraction of organs and three-dimensional detailed models of the human anatomy. All this is the basis for the construction of interactive simulators, to be used as a teaching aid in Medicine. The First International Conference on the Visible Human Project was held at the National Library of Medicine in Bethesda in October 1996.

The Cilea-MediSan section of the Cilea University Consortium for Information and Communication Technology and the Politecnico di Milano have investigated the difficulties in using 56 Gigabytes of data via Internet from the Continental Europe through transatlantic connections. Thus, the Politecnico di Milano and the Cilea have combined their technology and capacity to open the Milano Mirror Site of the VHD. This is intended to offer a high-level function to users of the Continental Europe, provided they are licensed by the National Library of Medicine to use the VHD data. Thanks to the sponsorships of four companies (Bracco, Imation, Hewlett-Packard and Telecom Italia) the "Visible Human Dataset - Milano Mirror Site" $\circledast$ opened its service to the Continental Europe at the http://vhd-mms.cilea.it on February 1997 and became fully operational in July 1997. The services, based on the new hardware, include FTP and other functions.

\section{Concluding Remarks}

The educational and research activities in Medical Informatics and Telemedicine described here are also available at http://www.medinfopoli. polimi.it.

Comments from the readers, as well as from colleagues are appreciated

We are aiming to establish cooper: tive initiatives, within the field of Med cal Informatics and Telemedicine, be, tween the Politecnico di Milano and other institutions, both in Italy and abroad. Specific items could includ student exchange, distance teachirf via videoconferencing systems, teach exchange, continuing education $\mathrm{pro}_{1}$ grams, and many other topics of poten tial interest.

\section{Acknowledgments}

The activities described here hav made me, happily, indebted to a num-1 ber of academic authorities, colleague collaborators, students and technicians Just the best treasure of a teacher is his students, I am grateful to so many of them. I have had the good fortune of co-authoring publications with them often at the international level; they are a vivid part of my scientific adventura Let me express special gratitude to the students of the Italian $\mathrm{PhD}$ program in Bioengineering who worked very close to me for several years. In chronologi? cal order they were Ruggero Rossi, Giuseppe Pozzi, Carlo Combi and Luis Portoni.

Address of the author:

Prof. Ing. Francesco Pinciroli, Chair of Medical Informatics, Dipartimento di Bioingegneria, Politecnico di Milano,

Piazza L. da Vinci, 32

20133 Milano, Italy

E-mail:pinciroli@biomed.polimi.it http://www.medinfopoli.polimi.it 